\title{
Does the Use of Injectable Atelocollagen during Arthroscopic Rotator Cuff Repair Improve Clinical and Structural Outcomes?
}

\author{
In Bo Kim, Eun Yeol Kim ${ }^{\varpi}$, Kuk Pil Lim, Ki Seong Heo \\ Department of Orthopedic Surgery, Busan Bumin Hospital, Busan, Korea
}

Background: Since the establishment of biological augmentation to improve the treatment of rotator cuff tears, it is imperative to explore newer techniques to reduce the retear rate and improve long-term shoulder function after rotator cuff repair. This study was undertaken to determine the consequences of a gel-type atelocollagen injection during arthroscopic rotator cuff repair on clinical outcomes, and evaluate its effect on structural integrity.

Methods: Between January 2014 and June 2015, 121 patients with full thickness rotator cuff tears underwent arthroscopic rotator cuff repair. Of these, 61 patients were subjected to arthroscopic rotator cuff repair in combination with an atelocollagen injection (group I), and 60 patients underwent arthroscopic rotator cuff repair alone (group II). The visual analogue scale (VAS) for pain and the Korean Shoulder Society (KSS) scores were evaluated preoperatively and postoperatively. Magnetic resonance imaging (MRI) was performed at 6 months postoperatively, to assess the integrity of the repair.

Results: VAS scores were significantly lower in group I than in group II at 3, 7, and 14 days after surgery. KSS scores showed no significant difference between groups in the 24 months period of follow-up. No significant difference was obtained in the healing rate of the rotator cuff tear at 6 months postoperatively $(p=0.529)$.

Conclusions: Although a gel-type atelocollagen injection results in reduced pain in patients at 2 weeks after surgery, our study does not substantiate the administration of atelocollagen during rotator cuff repair to improve the clinical outcomes and healing of the rotator cuff. (Clin Shoulder Elbow 2019;22(4):183-189)

Key Words: Atelocollagen; Rotator cuff repair; Clinical outcome; Tendon integrity

\section{Introduction}

Rotator cuff tear is a common shoulder disorder causing pain and functional disability, including weakness and decreased range of motion (ROM) in the shoulder joint, and is more prevalent in the elderly. ${ }^{1,2)}$ Although arthroscopic rotator cuff repair is a commonly accepted treatment widely used to obtain satisfactory results, ${ }^{3)}$ postoperative retear of the rotator cuff is still an important postoperative setback, since the risk of retear influences the rehabilitation process and quality of life in patients post-surgery.

Various surgical techniques that maintain firm intergradation at the bone-tendon interface have been applied to decrease the retear rate. ${ }^{5-7)}$ However, these strategies often fail due to the difficulty in achieving enthetic regeneration. In most cases, a fibrovascular and disorganized scar tissue is formed at the bonetendon interface. ${ }^{8)}$ Therefore, the goals of rotator cuff repair should be to provide a biomechanical and biological environment around the repair site to promote regeneration of the native insertion site, and to prevent the formation of scar tissue. ${ }^{9)}$

Several studies have investigated to improve the healing of repair and induce regeneration of functional tissues through biological strategies such as platelet rich plasma (PRP), mesenchymal stem cell (MSC), growth factors, and cytokines. PRP has been used to enhance tendon-bone healing and to decrease retear rate. ${ }^{10)}$ However, PRP enhancement after rotator cuff repair exerts no significant effect on the clinical and patient-reported

Received September 24, 2019. Revised October 28, 2019. Accepted October 30, 2019.

Correspondence to: Eun Yeol Kim

Department of Orthopedic Surgery, Busan Bumin Hospital, 59 Mandeok-daero, Buk-gu, Busan 46555, Korea

Tel: +82-51-330-3082, Fax: +82-51-330-3075, E-mail: coolkey102@naver.com, ORCID: https://orcid.org/0000-0003-3693-8749

IRB approval: Busan Bumin Hospital (No. 201909-BM_008).

Financial support: None. Conflict of interests: None. 
outcomes. ${ }^{11)}$ Injection of stem cells during rotator cuff repair are reported to significantly improve structural outcomes in terms of the retear rate. ${ }^{12)}$ Recent studies also demonstrate that biologic compounds, such as hyaluronic acid and atelocollagen, accelerate tendon to bone healing in the rabbit rotator cuff tear model, enhancing biomechanical strength and chondroid formation at the repair site. ${ }^{13,14}$

This current study aimed to investigate the efficacy of atelocollagen, which is used in arthroscopic rotator cuff repair for chronic tendinopathy of human. We hypothesized that a local injection of gel-type atelocollagen into the primary rotator cuff repair would result in improved tendon healing and better clinical outcomes.

\section{Methods}

\section{Patient Selection}

From January 2014 to June 2015, a total of 228 patients were identified, who subsequently underwent arthroscopic rotator cuff repair by the senior author. Only patients with a fullthickness tear of the supraspinatus tendon, with or without a combined full thickness tear of any other rotator cuff tendon, were included. The exclusion criteria included partial-thickness tear of the supraspinatus tendon regardless of full-thickness tears of the other cuff tendons, isolated tear of the subscapularis tendon, massive irreparable rotator cuff tear requiring alternative surgery such as arthroscopic bridging or interposition repair, advanced glenohumeral arthritis, biceps lesion requiring tenotomy or tenodesis, acromioclavicular arthritis requiring distal clavicle resection, revision rotator cuff surgery, previous shoulder surgery of any cause, and patients with ineligible magnetic resonance imaging (MRI) results from other institutes.

Of the 228 patients identified, 59 were excluded based on the above-mentioned criteria. An additional 48 patients were excluded as they had not undergone follow-up observation for at least 2 years, or had refused to undergo follow-up MRI. Ultimately, 121 patients were enrolled for this study; 61 patients were subjected to arthroscopic rotator cuff repair in combination with an atelocollagen injection (group I), and 60 patients underwent arthroscopic rotator cuff repair alone (group II). All patients were retrospectively reviewed for gathering the data.

\section{Surgical Procedures and Injectable Atelocollagen Application}

All patients underwent shoulder arthroscopic surgery in the lateral decubitus position under regional anesthesia with interscalene brachial plexus block. The arm was maintained at approximately $30^{\circ}$ abduction and $30^{\circ}$ forward flexion with $4 \mathrm{~kg}$ traction. Diagnostic glenohumeral arthroscopy was completed using a $30^{\circ}$ arthroscope through a standard posterior portal. Standard anterior and lateral portals were introduced via an outside-in technique. The torn supraspinatus with or without the infraspinatus was then debrided to confirm the margin and evaluate the mobility of the tendon. The tendon was released, if required, until the cuff was adequately mobilized. The tendon was then repaired to the prepared greater tuberosity with suture anchors. All patients were treated using the arthroscopic suture bridge technique. A standard acromioplasty was always done. At the end of the arthroscopy, the traction force was released, and withdrawal resistance of the repaired construct was tested by gently moving the arm.

We used gel-type $(3 \mathrm{ml})$ products of type I atelocollagen (RegenSeal; Sewon Cellontech Co., Seoul, Korea) derived from porcine dermal skin. Using the lateral portal, a spinal needle attached to the injectable gel-type atelocollagen-filled syringe was placed, without cannula, between the bone and the repaired rotator cuff. The inflow was then closed and the arthroscopic fluid was carefully aspirated via the outflow cannula. All other cannulas were removed, and the gel-type atelocollagen was slowly injected under arthroscopic visualization.

\section{Postoperative Protocol}

All patients adhered to the same rehabilitation protocol. Pendulum exercise and active elbow ROM exercise were started immediately after surgery, and passive forward flexion was started 1 day after surgery. Early passive ROM was permitted within a tolerable range. The shoulder was immobilized for 6 weeks using an abduction brace. Active assisted ROM exercises were started after 6 weeks. Patients began strengthening exercise after 3 months. Recreational activities or manual labor were permitted after 3 months, and full return to heavy manual work was allowed after 6 months.

\section{Clinical Assessment and Radiographic Evaluation}

All patients were evaluated clinically and radiographically before surgery and at follow-up. Demographic data collected for both groups include age, sex, symptom duration, mediolateral $(\mathrm{ML})$ and anteroposterior (AP) tear size of tendon, tendon involvement, degree of fatty infiltration of rotator cuff muscles, acromiohumeral distance (AHD), and tangent sign. Before undergoing arthroscopic rotator cuff repair, patients were subjected to MRI. The ML and AP size of tendon tears (in millimeters) were evaluated on T2-weighted images in oblique coronal and oblique sagittal MRI, as described by Davidson et al. ${ }^{15)}$ According to the tendon involvement, the patterns of tendon tear were classified into 4 types: supraspinatus tears (type 1), supraspinatus and subscapularis tears (type 2), supraspinatus and infraspinatus tears (type 3), and tears involving all 3 tendons (type 4). ${ }^{16)}$ Fatty infiltration of rotator cuff muscles was measured using preoperative MRI, according to the classification established by Goutallier et al. ${ }^{17)}$ and modified by Fuchs et al. ${ }^{18)}$ Muscle atrophy of the supraspinatus was evaluated by assessing the tangent sign on 
the most lateral T2-weighted oblique sagittal MRI in which the scapular spine contacted the scapular body. The tangent sign was considered positive when the superior border of the supraspinatus muscle belly was inferior in relation to the line tangential to the coracoids and scapular spine. ${ }^{19)}$ Clinical outcomes were evaluated preoperatively and at 3, 12, and 24 months after surgery, by applying the Korean Shoulder Society (KSS) score. All patients were evaluated for pain at 3, 7, and 14 days postoperatively, using the visual analogue scale (VAS). An MRI study was performed on all patients to evaluate the structural integrity, approximately 6 months after surgery. The criterion for retear was the lack of tendon continuity, as observed in one slice of the coronal plane. Retears were evaluated by MRI, using the Sugaya classification for patients. ${ }^{20)}$ All MRI studies were performed with the Magnetom Symphony 1.5T MRI system (Siemen, Erlangen, Germany) and the Signa EXCITE 1.5T MRI system (GE, Milwaukee, WI, USA), and were reviewed by 2 experienced radiologists.

\section{Statistical Analysis}

Independent t-test was performed to assess the difference of continuous variables between the two groups, including age, symptom duration before surgery, ML and AP tear sizes, AHD, VAS, and KSS. Chi-squared test or Fisher's exact test was used to compare the differences of nominal variables such as sex, tendon involvement, fatty infiltration, and prevalence of tangent sign. Paired t-test was implemented to compare preoperative and 14 days postoperative VAS in each group. IBM SPSS software package (ver. 20.0; IBM Corp., Armonk, NY, USA) was used for all statistical analyses, with the $\alpha$ level set at 0.05 .

\section{Results}

Group I comprised 61 patients (26 males and 35 females), with a mean age of $59.8 \pm 7.3$ years. Group II included 60 subjects (31 males and 29 females), with a mean age of 57.8 \pm 8.1 years. The mean follow-up duration was 26.7 months, and mean time for postoperative MRI was 6.4 months. Patient characteristics were comparable between both groups, and are summarized in Table 1. Considering the tear types, 56 patients in group I and 56 patients in group II had lesions affecting only the supraspinatus tendon; only 2 patients in group II had tendon involvement type 2 (supraspinatus and subscapularis); whereas 5 patients in group I and 2 patients in group II revealed tendon involvement type 3 (supraspinatus and infraspinatus). No significant difference was found between the two groups $(p=0.129)$. A higher degree of fatty degeneration of the subscapularis $(p=0.044)$ and infraspinatus $(p=0.013)$ was observed in group II. However, there was no comparative analysis of the effects of fatty degeneration with a small number of retear. There were no occurrences of infections, neurovascular complications, or anchor pullout in this study.

No significant difference was obtained in VAS scores for pain between group I and group II at baseline. The mean VAS score improved significantly in both groups: from $5.3 \pm 2.1$ to $1.2 \pm$ 1.0 in group I $(p=0.001)$, and $6.3 \pm 1.7$ to $3.2 \pm 1.7$ in group II $(p=0.005)$. Furthermore, VAS scores were significantly lower in group I than in group II at 3 days $(p=0.007), 7$ days $(p=0.003)$,

Table 1. Comparison of Demographic Data between Group I and Group II

\begin{tabular}{|c|c|c|c|}
\hline Variable & Group I ( $n=61)$ & Group II $(n=60)$ & $p$-value ${ }^{*}$ \\
\hline Sex, male:female & $26(42.6): 35(57.4)$ & $31(51.7): 29(48.3)$ & 0.319 \\
\hline Age (yr) & $59.8 \pm 7.3$ & $57.8 \pm 8.1$ & 0.150 \\
\hline Symptom duration (mo) & $20.5 \pm 45.3$ & $9.5 \pm 14.6$ & 0.075 \\
\hline Mediolateral tear size (mm) & $24.6 \pm 11.6$ & $24.3 \pm 13.5$ & 0.898 \\
\hline Anteroposterior tear size (mm) & $19.3 \pm 11.6$ & $19.6 \pm 11.9$ & 0.890 \\
\hline Tendon involvement, type 1:2:3 & $56: 0: 5$ & $56: 2: 2$ & 0.129 \\
\hline \multicolumn{4}{|l|}{ Goutallier stages, grade $0: 1: 2: 3: 4$} \\
\hline Subscapularis & $20: 37: 4: 0: 0$ & $17: 43: 0: 0: 0$ & $0.044^{\dagger}$ \\
\hline Supraspinatus & $6: 48: 7: 0: 0$ & $12: 41: 6: 0: 1$ & 0.257 \\
\hline Infraspinatus & $2: 44: 14: 0: 0$ & $12: 32: 13: 2: 1$ & $0.013^{\dagger}$ \\
\hline Acromiohumeral distance (mm) & $8.9 \pm 1.7$ & $10.1 \pm 7.1$ & 0.225 \\
\hline Tangent sign & $1(1.6)$ & $2(3.3)$ & 0.619 \\
\hline
\end{tabular}

Values are presented as number (\%), mean \pm standard deviation, or number only.

Group I: with a gel-type atelocollagen, Group II: conventional repair, type 1: supraspinatus, type 2: supraspinatus and subscapularis, type 3: supraspinatus and infraspinatus.

${ }^{*}$ Statistically significant difference $(p<0.05)$.

${ }^{\dagger}$ Statistical significances $(p<0.05)$ were tested by chi-squared or Fisher's exact test. 
and 14 days $(p=0.003)$ post-surgery (Table 2$)$.

Postoperative KSS showed an improvement compared to preoperative status in both groups. There was no significant difference in pain of KSS domains at 3, 12, and 24 months after surgery. In the 24 months period of follow-up, the KSS scores showed no significant difference between the groups, except for strength at 3 months after surgery, which was significantly higher in group I than in group II $(7.8 \pm 1.6$ and $6.9 \pm 1.6$, respectively; $p=0.003$ ) (Table 3).

MRI assessment to evaluate integrity of the repair after 6 months revealed retear in 7 patients (11.5\%) in group I and 4 patients $(6.7 \%)$ in group II. Although the retear rate was higher in group I, the difference was statistically not significant $(p=0.529)$.

\section{Discussion}

Our data verifies that intraoperative administered gel-type atelocollagen leads to limited pain reduction, but is not associated with better clinical outcome within 2 years after surgical repair.

Patients in group I reported significantly better results, especially for 2 weeks postoperative pain. Pain relief allows for faster mobilization and improved function. The reduction of pain after atelocollagen injection was similar to that observed after a subacromial ropivacaine or PRP injection. ${ }^{21)}$ Previous studies show that autologous PRP reduces pain in the initial postoperative months and may be related to platelet analgesic properties by releasing protease activated receptor 4 peptides. ${ }^{22)} \mathrm{A}$ possible explanation for our result is related to that fact that atelocollagen is believed to have analgesic properties similar to PRP in the early stages of surgery. Another explanation for eventual tendon degeneration and inferior tendon tissue quality is an increase in inflammation." However, atelocollagen shows anti-inflammatory effect in the early tendon healing stage after cuff repair. ${ }^{14)}$ It is also believed that decreased pain is due to reduced release of pain causing factors in the early stage after surgery. Frequently, the clinical use of injectable atelocollagen results in more pain after injection than with other injections. Usually, pain begins at the commencement of the injection, and patients complain of the maximum pain during the first week. We need to consider that the difference observed in our current study may have arisen because while the clinical injection is usually applied in the subacromial space, our atelocollagen injection was administered between the tendon and bone. Numerous factors are known to affect early surgical pain, such as operation time, degree of soft tissue release or acromioplasty, and whether preoperatively frozen or not. The effect of applying injectable atelocollagen reduces early postoperative pain cannot be justified with our data, and requires clarification with further studies.

In our study, a final follow-up of 24 months after surgery shows significant improvement in both groups as compared with the preoperative phase, with no difference being observed between groups. The only different clinical results between the two groups were strength of the KSS score at 3 months after surgery. It is difficult to give clinical significance to this finding. At a minimum of 24 months of follow-up period, surgical repair of the rotator cuff tears resulted in significant clinical and structural improvements in both groups, independent of the use of atelocollagen. The main finding of this study is that gel-type atelocollagen injection in rotator cuff repair does not result in significantly improved shoulder function or structural consequences, when compared to arthroscopic repair without atelocollagen injection.

Recently, studies on improving the clinical outcome of rotator cuff tear treatment have been applied to biological augmentation as rotator cuff repair. Randelli et al. ${ }^{21)}$ reported that PRP injection significantly improves all clinical outcomes compared to control group at the 3-month follow-up, but no significant differences are observed at 6, 12, and 24 months. Flury et al. ${ }^{11)}$ reported that patients administered pure PRP show no significant improvement in functions at 3, 6, and 24 months after arthroscopic repair compared with control patients receiving ropivacaine. Kim et al. ${ }^{12)}$ treated 182 patients with rotator cuff tears using conventional repair technique plus MSC; they report that the Constant and University of California, Los Angeles (UCLA) scores improve after surgery, but there were no significant differences compared to conventional repair without MSC at the final follow-up.

The main finding of our study is that application of gel-type atelocollagen during the repair of rotator cuff tears does not significantly improve the structural outcomes in terms of the retear rate, when compared with conventional repair without a geltype atelocollagen injection.

Numerous biomaterials are being used to enhance the heal-

Table 2. Comparison of the Visual Analogue Scale (VAS) between Group I and Group II

\begin{tabular}{cccccc}
\hline VAS & Preoperative & $3 \mathrm{~d}$ & $7 \mathrm{~d}$ & $14 \mathrm{~d}$ & $p$-value $(0 \mathrm{~d}$ vs. $14 \mathrm{~d})$ \\
\hline Group I & $5.3 \pm 2.1$ & $2.7 \pm 1.9$ & $2.0 \pm 1.2$ & $1.2 \pm 1.0$ & $0.001^{*}$ \\
Group II & $6.3 \pm 1.7$ & $5.7 \pm 2.3$ & $4.1 \pm 2.7$ & $3.2 \pm 1.7$ & $0.005^{*}$ \\
\hline -value & 0.363 & $0.007^{*}$ & $0.003^{*}$ & $0.003^{*}$ & \\
\hline
\end{tabular}

Values are presented as mean \pm standard deviation.

Group I: with a gel-type atelocollagen, Group II: conventional repair.

*Statistically significant difference $(p<0.05)$. 
Table 3. Comparison of Clinical Outcomes between Group I and Group II

\begin{tabular}{|c|c|c|c|c|}
\hline KSS score & Preoperative & $3 \mathrm{mo}$ & $12 \mathrm{mo}$ & $24 \mathrm{mo}$ \\
\hline \multicolumn{5}{|l|}{ Function } \\
\hline Group I & $19.2 \pm 4.8$ & $23.0 \pm 4.2$ & $25.1 \pm 3.5$ & $26.3 \pm 2.8$ \\
\hline Group II & $19.3 \pm 4.2$ & $23.9 \pm 2.9$ & $25.1 \pm 4.2$ & $26.8 \pm 3.5$ \\
\hline$p$-value & 0.929 & 0.199 & 0.990 & 0.763 \\
\hline \multicolumn{5}{|l|}{ Pain } \\
\hline Group I & $13.1 \pm 3.3$ & $15.0 \pm 3.2$ & $15.5 \pm 2.4$ & $16.7 \pm 4.1$ \\
\hline Group II & $14.0 \pm 2.4$ & $15.3 \pm 2.2$ & $16.4 \pm 2.7$ & $17.6 \pm 2.6$ \\
\hline$p$-value & 0.103 & 0.606 & 0.197 & 0.499 \\
\hline \multicolumn{5}{|l|}{ Satisfaction } \\
\hline Group I & $4.5 \pm 2.7$ & $6.6 \pm 1.6$ & $7.2 \pm 1.1$ & $7.2 \pm 1.2$ \\
\hline Group II & $3.8 \pm 1.7$ & $6.5 \pm 1.5$ & $7.4 \pm 1.3$ & $7.9 \pm 1.5$ \\
\hline$p$-value & 0.124 & 0.747 & 0.563 & 0.260 \\
\hline \multicolumn{5}{|l|}{ ROM } \\
\hline Group I & $15.4 \pm 3.5$ & $14.0 \pm 3.1$ & $15.4 \pm 2.3$ & $17.3 \pm 1.0$ \\
\hline Group II & $14.0 \pm 4.1$ & $14.4 \pm 2.6$ & $16.7 \pm 2.7$ & $16.9 \pm 2.7$ \\
\hline$p$-value & 0.051 & 0.383 & 0.054 & 0.701 \\
\hline \multicolumn{5}{|l|}{ Strength } \\
\hline Group I & $5.9 \pm 2.6$ & $7.8 \pm 1.6$ & $9.1 \pm 1.1$ & $9.3 \pm 1.0$ \\
\hline Group II & $5.8 \pm 2.9$ & $6.9 \pm 1.6$ & $8.9 \pm 1.3$ & $9.3 \pm 1.2$ \\
\hline$p$-value & 0.833 & $0.003^{\star}$ & 0.574 & 0.945 \\
\hline \multicolumn{5}{|l|}{ Endurance } \\
\hline Group I & $5.0 \pm 4.7$ & $5.2 \pm 4.5$ & $8.0 \pm 3.8$ & $9.5 \pm 1.2$ \\
\hline Group II & $4.6 \pm 4.7$ & $4.1 \pm 4.3$ & $7.9 \pm 4.0$ & $9.4 \pm 2.4$ \\
\hline$p$-value & 0.690 & 0.165 & 0.935 & 0.933 \\
\hline \multicolumn{5}{|l|}{ Total } \\
\hline Group I & $63.0 \pm 15.1$ & $71.6 \pm 12.9$ & $80.1 \pm 9.4$ & $86.3 \pm 7.3$ \\
\hline Group II & $61.5 \pm 15.2$ & $70.8 \pm 9.0$ & $82.3 \pm 11.2$ & $88.0 \pm 8.9$ \\
\hline$p$-value & 0.583 & 0.726 & 0.443 & 0.686 \\
\hline
\end{tabular}

Values are presented as mean \pm standard deviation.

KSS: Korean Shoulder Society, ROM: range of motion, Group I: with a gel-type atelocollagen, Group II: conventional repair.

${ }^{\star}$ Statistically significant difference $(p<0.05)$.

ing of the tendon-to-bone interface for rotator cuff repair. The effect of stem cells on tendon healing may improve through targeted delivery of these cells to the repair site, and by appropriate modulation of the healing environment, including growth factors, cytokines, cell concentrations, and mechanical stimuli. ${ }^{23}$ Transforming growth factor-beta (TGF- $\beta$ ) is a crucial signal in the inflammatory and healing stages of tissue repair; TGF- $\beta$ has demonstrated promise in accelerating the healing of rotator cuff tendons and is reported to improve repairs in terms of histology and biomechanics. ${ }^{24)}$ Recombinant platelet derived growth factor (PDGF) is also shown to improve the biomechanics of repairs and increase bone-tendon interface when used with a collagen matrix. $^{25)}$

Tendon-to-bone healing occurs by a three-step repair process: inflammation, repair, and remodeling. The scar undergoes remodeling from type III collagen to type I collagen during the final remodeling phase. ${ }^{26)}$ Focusing on the role of collagen in the tendon healing process, several studies demonstrate its application for tendon repair, and the effect of collagen on tissue regeneration and healing. Stopak et al. ${ }^{27)}$ evaluated that type I collagen was added to develop buds of chicken feathers; it was found that the addition of collagen was incorporated into 
the normal connective tissue of feathers. This result shows that collagen is easily assimilated into the surrounding area, without any defects in the actual function of the body part. A study by Oliveira et al. ${ }^{28)}$ reports developing a type I collagen sponge to be used as a cartilage template scaffold. They found that collagen sponges mechanically support the cartilage templates in addition to biochemically allowing cell migration through sponge pores, without cell phenotype or differentiation changes. Moshiri et al. ${ }^{29)}$ report on implanted bovine type I collagen into a completely cleaved rabbit Achilles tendon; the collagen implant was entirely absorbed, and the regenerating tissue filled the injury at the end of the healing phase. They also noticed that the collagen implants attract other substances such as growth factors and fibroblasts to improve the healing process.

The numerous disadvantages of collagen-based scaffolds need to be considered in tendon and ligament substitutes such as cost, incompatibility, immunogenicity and disease transmission risks. A collagen molecule has an amino acid sequence (called telopeptide) at both the $\mathrm{N}$ - and C-terminals, which confers most of the antigenicity. Atelocollagen obtained by protease or pepsin treatment has a highly biocompatible triple helical structure, and possesses the lowest immunogenicity for complete removal of the collagen telopeptide. ${ }^{30)}$ Highly purified atelocollagen has many advantages for biocompatibility, and optimizes the collagen-cell interactions to minimize side effects. Atelocollagens are available as 2 types of products: gel and patch. A recent study by Suh et al. ${ }^{14)}$ reported that both histological and biomechanical studies demonstrate better results in an experimental group using patch-type atelocollagen in a rabbit model of the supraspinatus tendon tear. Atelocollagen provides an abundant site of adherent cells in the repair scaffold, and promotes tissue embolic neovascularization by activating cells and PRP. ${ }^{14)}$

The present study has some limitations. First, since this is not a blinded study, no definitive conclusion was reached due to research variability. Second, our study was a retrospective study with inherent limitations; hence, no meta-analysis could be performed to determine whether a true difference exists between healed rotator cuff and retear of rotator cuff. Third, this study was performed using a gel-type atelocollagen. If we used a patch-type atelocollagen as in the previous animal experiments, it is possible that we could have had different results. However, to the best of our knowledge, the strength of this study remains that it is the first attempt to compare the results of arthroscopic rotator cuff repair with or without atelocollagen injection, using both clinical and imaging criteria.

\section{Conclusion}

Although gel-type atelocollagen injection results in decreased pain at 2 weeks after surgery, our study does not support the use of a gel-type atelocollagen injection in arthroscopic rotator cuff repair to improve healing of rotator cuffs. We are unable to demonstrate superior clinical or structural outcomes of arthroscopic rotator cuff repair with atelocollagen as compared to without atelocollagen. Further studies are required to investigate the clinical outcomes of atelocollagen application.

\section{References}

1. Fehringer EV, Sun J, VanOeveren LS, Keller BK, Matsen FA 3rd. Full-thickness rotator cuff tear prevalence and correlation with function and co-morbidities in patients sixty-five years and older. J Shoulder Elbow Surg. 2008;17(6):881-5. doi: 10.1016/j.jse.2008.05.039.

2. Galatz LM, Ball CM, Teefey SA, Middleton WD, Yamaguchi K. The outcome and repair integrity of completely arthroscopically repaired large and massive rotator cuff tears. J Bone Joint Surg Am. 2004;86(2):219-24. doi: 10.2106/00004623200402000-00002.

3. Jost B, Pfirrmann CW, Gerber C, Switzerland Z. Clinical outcome after structural failure of rotator cuff repairs. J Bone Joint Surg Am. 2000;82(3):304-14. doi: 10.2106/00004623200003000-00002.

4. Oh JH, Kim SH, Ji HM, Jo KH, Bin SW, Gong HS. Prognostic factors affecting anatomic outcome of rotator cuff repair and correlation with functional outcome. Arthroscopy. 2009;25(1):30-9. doi: 10.1016/j.arthro.2008.08.010.

5. Anderson K, Boothby M, Aschenbrener D, van Holsbeeck M. Outcome and structural integrity after arthroscopic rotator cuff repair using 2 rows of fixation: minimum 2-year followup. Am J Sports Med. 2006;34(12):1899-905. doi: 10.1177/ 0363546506290187

6. Frank JB, ElAttrache NS, Dines JS, Blackburn A, Crues J, Tibone JE. Repair site integrity after arthroscopic transosseousequivalent suture-bridge rotator cuff repair. Am J Sports Med. 2008;36(8):1496-503. doi: 10.1177/0363546507313574.

7. Ficklscherer A, Loitsch T, Serr M, et al. Does footprint preparation influence tendon-to-bone healing after rotator cuff repair in an animal model? Arthroscopy. 2014;30(2):188-94. doi: 10.1016/j.arthro.2013.11.016.

8. Boileau P, Brassart N, Watkinson DJ, Carles M, Hatzidakis AM, Krishnan SG. Arthroscopic repair of full-thickness tears of the supraspinatus: does the tendon really heal? J Bone Joint Surg Am. 2005;87(6):1229-40. doi: 10.2106/JBJS.D.02035.

9. Kovacevic D, Rodeo SA. Biological augmentation of rotator cuff tendon repair. Clin Orthop Relat Res. 2008;466(3):62233. doi: 10.1007/s11999-007-0112-4.

10. Jo CH, Shin JS, Shin WH, Lee SY, Yoon KS, Shin S. Plateletrich plasma for arthroscopic repair of medium to large rotator cuff tears: a randomized controlled trial. Am J Sports Med. 2015;43(9):2102-10. doi: 10.1177/0363546515587081.

11. Flury M, Rickenbacher D, Schwyzer HK, et al. Does pure 
platelet-rich plasma affect postoperative clinical outcomes after arthroscopic rotator cuff repair? A randomized controlled trial. Am J Sports Med. 2016;44(8):2136-46. doi: 10.1177/ 0363546516645518.

12. Kim YS, Sung CH, Chung SH, Kwak SJ, Koh YG. Does an injection of adipose-derived mesenchymal stem cells loaded in fibrin glue influence rotator cuff repair outcomes? A clinical and magnetic resonance imaging study. Am J Sports Med. 2017;45(9):2010-18. doi: 10.1177/0363546517702863.

13. Honda H, Gotoh M, Kanazawa T, et al. Hyaluronic acid accelerates tendon-to-bone healing after rotator cuff repair. Am J Sports Med. 2017;45(14):3322-30. doi: 10.1177/0363546517720199.

14. Suh DS, Lee JK, Yoo JC, et al. Atelocollagen enhances the healing of rotator cuff tendon in rabbit model. Am J Sports Med. 2017;45(9):2019-27. doi: 10.1177/0363546517703336.

15. Davidson JF, Burkhart SS, Richards DP, Campbell SE. Use of preoperative magnetic resonance imaging to predict rotator cuff tear pattern and method of repair. Arthroscopy. 2005;21(12):1428. doi: 10.1016/j.arthro.2005.09.015.

16. Kim IB, Kim MW. Risk factors for retear after arthroscopic repair of full-thickness rotator cuff tears using the suture bridge technique: classification system. Arthroscopy. 2016;32(11): 2191-200. doi: 10.1016/j.arthro.2016.03.012.

17. Goutallier D, Postel JM, Bernageau J, Lavau L, Voisin MC. Fatty muscle degeneration in cuff ruptures. Pre- and postoperative evaluation by CT scan. Clin Orthop Relat Res. 1994;(304):7883. doi: 10.1097/00003086-199407000-00014.

18. Fuchs B, Weishaupt D, Zanetti M, Hodler J, Gerber C. Fatty degeneration of the muscles of the rotator cuff: assessment by computed tomography versus magnetic resonance imaging. J Shoulder Elbow Surg. 1999;8(6):599-605. doi: 10.1016/ s1058-2746(99)90097-6.

19. Mellado JM, Calmet J, Olona M, et al. Surgically repaired massive rotator cuff tears: MRI of tendon integrity, muscle fatty degeneration, and muscle atrophy correlated with intraoperative and clinical findings. AJR Am J Roentgenol. 2005;184(5):145663. doi: 10.2214/ajr.184.5.01841456.

20. Sugaya H, Maeda K, Matsuki K, Moriishi J. Functional and structural outcome after arthroscopic full-thickness rotator cuff repair: single-row versus dual-row fixation. Arthroscopy. 2005;21(11):1307-16. doi: 10.1016/j.arthro.2005.08.011.

21. Randelli P, Arrigoni P, Ragone V, Aliprandi A, Cabitza P. Platelet rich plasma in arthroscopic rotator cuff repair: a prospective RCT study, 2-year follow-up. J Shoulder Elbow Surg. 2011; 20(4):518-28. doi: 10.1016/j.jse.2011.02.008.

22. Asfaha S, Cenac N, Houle S, et al. Protease-activated receptor-4: a novel mechanism of inflammatory pain modulation. $\mathrm{Br}$ J Pharmacol. 2007;150(2):176-85. doi: 10.1038/s.bjp.0706975.

23. Gulotta LV, Chaudhury S, Wiznia D. Stem cells for augmenting tendon repair. Stem Cells Int. 2012;2012:291431. doi: 10.1155/2012/291431.

24. Kovacevic D, Fox AJ, Bedi A, et al. Calcium-phosphate matrix with or without TGF- $\beta 3$ improves tendon-bone healing after rotator cuff repair. Am J Sports Med. 2011;39(4):811-9. doi: 10.1177/0363546511399378.

25. Hee CK, Dines JS, Dines DM, et al. Augmentation of a rotator cuff suture repair using rhPDGF-BB and a type I bovine collagen matrix in an ovine model. Am J Sports Med. 2011;39(8):1630-9. doi: 10.1177/0363546511404942.

26. Weeks KD 3rd, Dines JS, Rodeo SA, Bedi A. The basic science behind biologic augmentation of tendon-bone healing: a scientific review. Instr Course Lect. 2014;63:443-50.

27. Stopak D, Wessells NK, Harris AK. Morphogenetic rearrangement of injected collagen in developing chicken limb buds. Proc Natl Acad Sci U S A. 1985;82(9):2804-8. doi: 10.1073/ pnas.82.9.2804.

28. Oliveira SM, Ringshia RA, Legeros RZ, et al. An improved collagen scaffold for skeletal regeneration. J Biomed Mater Res A. 2010;94(2):371-9. doi: 10.1002/jbm.a.32694.

29. Moshiri A, Oryan A, Meimandi-Parizi A. Role of tissue-engineered artificial tendon in healing of a large Achilles tendon defect model in rabbits. J Am Coll Surg. 2013;217(3):421-41. e8. doi: 10.1016/j.jamcollsurg.2013.03.025.

30. Lynn AK, Yannas IV, Bonfield W. Antigenicity and immunogenicity of collagen. J Biomed Mater Res B Appl Biomater. 2004;71(2):343-54. doi: 10.1002/jbm.b.30096. 